\title{
An Unsupervisory Qualitative Image Enhancement using Adaptive Morphological Bilateral Filter for Medical Images
}

\author{
B.Sridhar \\ Professor \\ Dept. of ECE \\ Lendi Institute of Engg. \& Tech. \\ Vizianagaram, India
}

\author{
K.V.V.S.Reddy, PhD \\ Professor \\ Dept. of ECE \\ Andhra University \\ Visakhapatnam, India
}

\author{
A.M.Prasad, PhD \\ Professor \\ Dept. of ECE \\ JNT University \\ Kakinada, India
}

\begin{abstract}
The Adaptive bilateral filter is able to smooth the noise, while enhancing edges and textures in the image. Morphology operations such as dilation, erosion, opening and closing are offering a quality Sharpening enhancement. The performance of the filter is to be improved by including the mathematical morphology operations along with adaptive bilateral filter process. The parameters of the Adaptive bilateral filter are optimized with a training procedure. Adaptive bilateral filter is removing noise from the images gives significantly sharper than those restored by the bilateral filter. The present proposed method is the modified algorithm of an adaptive bilateral filter with mathematical morphology operations for medical images. The proposed algorithm applied to various types of medical images and deserved results are obtained. The performance analysis of the filter with respective design parameters and metrics are compared with existed algorithm and relative graphs are depicted.
\end{abstract}

\section{Keywords}

Medical images, adaptive bilateral filtering, mathematical morphology, PSNR, MSE, stopping time

\section{INTRODUCTION}

The bilateral filtering gives a promising result in the area of image de nosing. It is becoming famous filter not only useful for removal of noise but also using for image segmentation, image registration, image interpolation and video processing etc which is pointed out by a quite lot of researchers[17,21]. Some of the scholars given a complete theoretical analysis of the bilateral filter and connected with the general approaches, the modified filter is applied to remove the noise from the image [10].

Buyue Zhang et.al [1] developed the adaptive bilateral filter $(\mathrm{ABF})$ is shown better performance than the ordinary form of the bilateral filters. This filter is modified with two important changes. One, an offset value is included along with range filter. Next both width and range of the adaptive filters are adaptive with respective output value. The output of the edge preserved with Laplacian of Gaussian (LOG) operation is improved the sharpness. The working methodology is purely depending on the image structure. The proposed method is shown MSE between the original and reconstructed images is minimized for each class of pixels.
Alexander Wong [2] introduced a different perceptually based process for noise removal of image signals represented by low signal to noise ratios. Results are shown that the process is effective at removing signal noise, while enhancing both qualitatively and quantitatively. Jinshan Tang et.al [3] developed a new bilateral filter based method to reduce speckle noise reduction in ultrasound images for the purpose of segmentation and Measurement,

H.W. Kang et.al [4] introduced an adaptive bilateral filter to preserve the curve strokes and reduced superimpose noise on the image. This method is make easy automatic generation of artistic illustration in an order to adaptive guide the curved stroke directions based on the importance map. The experimental results shown that scheme facilitates automatic production of artistic illustrations in a broad range of rendering styles.

Danny Barash et.al [5] shown the relationship between bilateral filtering and anisotropic diffusion been examined. The method proved that adaptive bilateral filter is given the performance nearly equal nonlinear filters discussed in image processing smoothing techniques. They provide a connection between the anisotropic and bilateral filters.

Mathematical morphology is one of the emerging areas in image processing. This is quite popular because it offers a simple set of operations technically called dilation, erosion, remain operations is a combination of these operations [11]. Yoshinori ITO [6] proposed an impulse noise removal filter using mathematical morphology. Xiaoping Linet.al [7] given attempts on Mathematical Morphology techniques is implementation process based on SCILAB toolbox. The toolbox is open source software and makes connection with mathematical morphology tool box. Joseph M. Reinhardt et.al [8] were described an adaptable search-based approach for morphological Shape enhancement. This method also used other applications purpose such as image recognition, coding, object detection. Patrick Guidotti [9] proposed a fourth order diffusion partial differential equations (PDE) is proposed and investigated, a smoothing technique also proposed by them to reduces processing time and improves the performance of this model. B.Sridhar et.al [12] presented the performance analysis of adaptive bilateral filter is evaluated by pixel to noise ratio and mean square errors. The performed values are calculated by changing the parameters of the adaptive filter half width values and standard deviations 
The proposed method is the adaptive bilateral filtering along with mathematical operations for removal noise effecting at low frequency pixel [3]. Here it is applied on the medical images, where it is necessary preserving the shapes and removing the noises. Here we applied a series of operations opening and closing of morphology; there after an adaptive bilateral filter is applied with vary the width and range of the filters. In order to improvement of the sharpness Laplacian of Gaussian (LOG) is included.

The remaining sections of the paper are organized as follows. In section 2, a review of the necessary background required to effectively implement our algorithm is presented. The proposed algorithm is described in Section 3. Experimental results and discussions of the proposed algorithm are discussed in section 4, and conclusions are drawn in section 5 . ask that authors follow some simple guidelines. In essence, we ask you to make your paper look exactly like this document. The easiest way to do this is simply to download the template, and replace the content with your own material.

\section{BACKGROUND}

\subsection{Mathematical morphology (MM)}

The most basic morphological operations are dilation and erosion. Dilation adds pixels to the boundaries of objects in an image, while erosion removes pixels on object boundaries. The number of pixels added or removed from the objects in an image depends on the size and shape of the structuring element used to process the image. In the morphological dilation and erosion operations, the state of any given pixel in the output image is determined by applying a rule to the corresponding pixel and its neighbors in the input image. The rule used to process the pixels defines the operation as dilation or erosion [13].

\subsection{Understanding Structuring Elements}

An essential part of the dilation and erosion operations is the structuring element(SE) is used to probe the input image. A structuring element is a matrix consisting of only 0's and 1's that can have any arbitrary shape and size [7]. The pixels with values of 1 define the neighborhood. Two-dimensional, or flat, structuring elements are typically much smaller than the image being processed. The center pixel of the structuring element, called the origin, identifies the pixel of interest or the pixel being processed. The pixels in the structuring element containing 1's define the neighborhood of the structuring element. These pixels are also considered in dilation or erosion processing. The accretion of $i$, then defining to different features from the various values of image the size of the structure must be adjusted. In general, select basic structure element shape, and the no of structure elements of the order $(2 i+1) X(2 i+1)$.

The two basic morphological operations, dilation and erosion with a structuring element $\mathrm{B}$, are defined for a grey-scale image $f \in L \alpha$ (IR2) by [1]

$$
\begin{aligned}
& \text { Dilation: }(f \bigoplus B)(x)=\sup \{f(x-y), y \in B\} \\
& \text { Erosion: }(f \Theta B)(x)=\inf \{f(x+y), y \in B\}
\end{aligned}
$$

These names can be easily motivated when considering a shape in a binary image and a disc shaped structuring element. In this case dilation blows up its boundaries, while erosion shrinks them. Dilation and erosion form the basis for constructing other morphological processes, for instance opening and closing:

$$
\begin{aligned}
& \text { Opening: }(f \circ B)(x)=((f \Theta B) \oplus B)(x), \\
& \text { Closing: }(f \bullet B)(x)=((f \oplus B) \Theta B)(x) .
\end{aligned}
$$

In the preceding shape interpretation opening smoothes the shape by breaking narrow is the uses and eliminating small islands, while closing smoothes by eliminating small holes.

\subsection{Selection of structure elements (SE)}

Structure elements are classified in to Omni directional and multi scaled elements is one way to plot out square windows [20]. In general consider $\alpha$ is the angle of rotation. Let us consider $\mathrm{N}$ is a value then order of the SE is $(2 \mathrm{~N}+1) \mathrm{X}$ $(2 \mathrm{~N}+1)$. The equation of the structure element is represented as

$$
W=\left\{\frac{s(n 1+s 1 n 2+s 2)}{\theta s}=s \alpha-N \leq s 1, s 2 \ll N\right.
$$

For all $s=0,1--4 \mathrm{~N}-1$ and $\alpha$ is the angle of the rotation expressed as $\alpha=180^{\circ} / 4 \mathrm{~N}$. Suppose $\mathrm{N}=2$ then dimension of the $\mathrm{SE}$ is $5 \mathrm{x} 5$, the angle value is obtained as $\alpha=0,22.5$, $45,135,157.5$ Consider the structure element sequence have same shape in common and size increased follow

\subsection{Adaptive bilateral filtering}

Bilateral filtering smoothes images while preserving edges, by means of a nonlinear combination of nearby image values. The method is non-iterative, local, and simple. It combines gray levels or colors based on both their geometric closeness and their photometric similarity, and prefers near values to distant values in both domain and range. The bilateral filter proposed by Tomasi and Manduchi in 1998 is a nonlinear filter that smoothes the noise while preserving edge structures [1]. Bilateral filter are a spatial domain filter, the response of the filter is given in the equation (5)

$$
y(m . n)=\sum_{k} \sum_{l} h[m, n ; k, l] x[k, l]
$$

$\mathbf{Y}(\mathbf{m}, \mathbf{n})$ is the noise removed image.

$h[m o, n o ; k, l]$ is the response at $[\mathrm{m}, \mathrm{n}]$ to an impulse $\mathrm{I}[\mathrm{k}, \mathrm{l}]$ and $x[\mathrm{~m}, \mathrm{n}]$ is the degraded image.

Where $(m o, n o)$ is the center pixel of the window $\Omega_{m_{0}, n_{0}}$.

$\sigma_{\mathrm{d}}$ and $\sigma_{\mathrm{r}}$ are the standard deviations of the domain and range Gaussian filters, respectively

$r\left(m_{0}, n_{0}\right)=$
$\sum_{m=m 0-N}^{m o+N} \sum_{n=n o-N}^{n o+N} e^{\left(\frac{\left(m-m_{0}\right)^{2}+\left(n-n_{0}\right)^{2}}{2 \sigma_{d}^{2}}\right)} \cdot e^{-\frac{1}{2}\left(\frac{g[m, n]-g\left[m_{0}, n_{0}\right]-w\left[m_{0}, n_{0}\right]}{\sigma_{r}\left[m_{0}, n_{0}\right]}\right)_{2}}$

$h\left[m, n ; m_{0}, n_{0}\right]=$
$I\left(\Omega_{m_{0}, n_{0}}\right) r_{m_{0}, n_{0}}^{-1} e^{\left(\frac{\left(m-m_{0}\right)^{2}+\left(n-n_{0}\right)^{2}}{2 \sigma_{d}{ }^{2}}\right)} \cdot e^{-\frac{1}{2}\left(\frac{g[m, n]-g\left[m_{0}, n_{0}\right]-w\left[m_{0}, n_{0}\right]}{\sigma_{r}\left[m_{0}, n_{0}\right]}\right)^{2}}$

$\mathrm{r}\left(\mathrm{m}_{\mathrm{o}}, \mathrm{n}_{\mathrm{o}}\right)$ is a normalization factor that assures that the filter preserves average gray value in constant areas of the image. 
A Gaussian filter is filtering the low frequency noise and restores the edges. Combinations of domain and range Gaussian filters are applied here to give maximum weight pixels near to centre value. A combined operations of domain and range filter along with the bilateral filter at nearer to edge pixel gray level values is become stretched out Gaussian filter is slopping around the edge. This guarantee takes an average of adjacent pixel values and minimizes the gradient direction. Thus, the bilateral filter greatly smooth's the noise and restoring edge formations.

The ABF maintains the actual form of bilateral filter [16], In addition two significant modifications is included. As shown in the equation $6 \& 7$ is consists two exponential functions one is the operator of range filters and second the domain filter functions. The range filters is included an offset(w) function and width is introduced in domain filters. These gives the bilateral filter is spatially adaptive. If the offset value is zero and width is constant the ABF is acts as an ordinary bilateral filter. The variation of these two values or either one is fixed the filters shows very good properties to restores the image and edges are sharpen. It is concentrated on edges at maximum level and improves the slope.

In $\mathrm{ABF}$ the pixel gray level variation plays an important role during the training of filter. Here we choose the difference between the centre pixel value and mean of the local window. Its response shows more effect on the strength of edges, separates the regions and reduces the robustness to noise.
Therefore Laplacian of Gaussian is applied to the image before being submitted to the filtering operation.

\section{PROPOSED METHOD}

Gray level medical images are considered for the proposed system. Two approaches are followed; one for extracting the boundary and the other for noise removal through adaptive bilateral filter.

The block diagram of the proposed method is shown in fig.1. Initially selected medical images are loaded into MATLAB environment and convert the gray scale values into Double precision format and normalize them in the interval $[0,1]$. Later these pixel values are blurred at certain level before the filtering process. Further histograms are displayed to identify the presence of noises. There after a series of mathematical morphological operation techniques like opening by reconstruction, opening, closing and closing by reconstruction are applied to the medical images in order[18] to mark the valley and peak intensities of the gray scale values. Finally complement of the image is done to get a marked image. Now set the bilateral filter parameters like half-width and domain range of filter in terms of a standard deviation values and apply the adaptive bilateral filtering operation. Obtain the resulted image and its contrast enhancement values through histogram. Now calculate the performance metrics of the filter like the Mean Squared Error, PSNR, principal correlation coefficient and time etc.

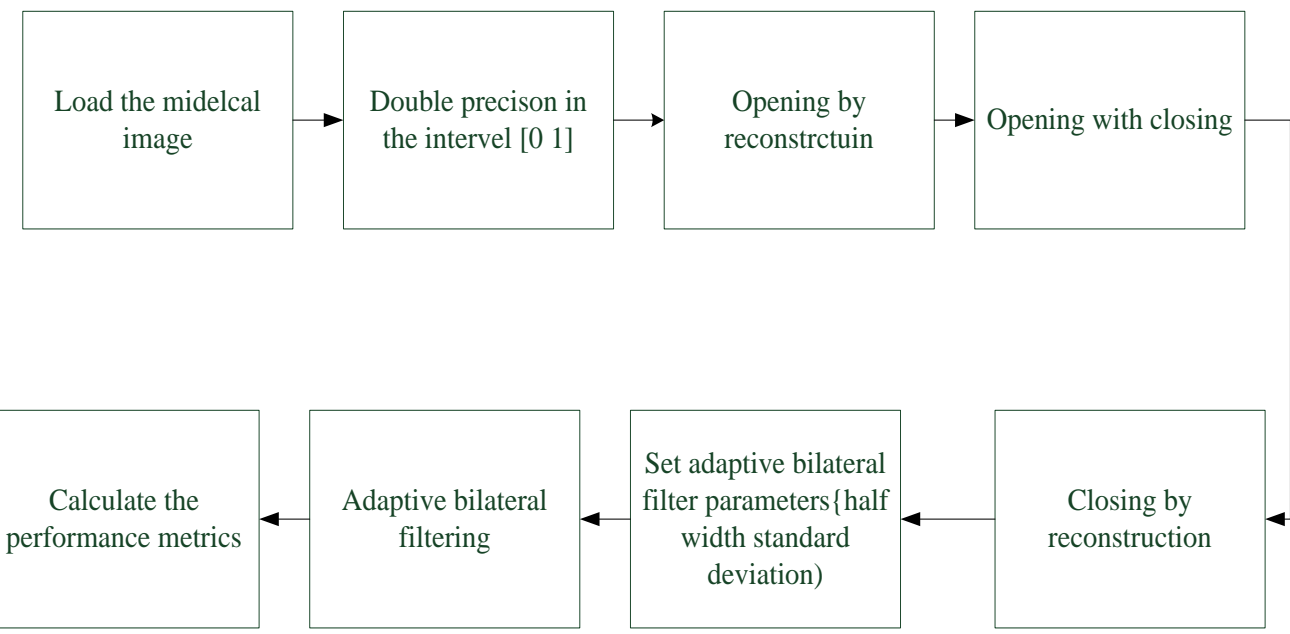

Fig 1: Block diagram of the proposed method

\section{RESULTS AND DISCUSSIONS}

All medical images are represented as gray scale images. Every pixel in gray scale images arise relative to the intensity or gray value. The variation of the intensity appears in dark and light regions of the image related to the valleys and domes respectively. The main challenge in medical image analysis is to preserve the regions with edges and remove the existing robust noise in the images. For Example if we consider the mammograms [15] having complex intensity values with around tissues. Regional maxima value can have the important feature. So we can mark the area to preserve important information. In order to preserve this information mathematical operations are used. Unless otherwise noted, all experiments are performed on 256 pixel square images. This phenomenon is illustrate in Fig. 1 on a test image with $30 \%$ random noise, de-noised with two types of structure elements, one is a multi structure elements of circular (disc) shape and dimensions $3 \times 3$. The parameters of the $\mathrm{ABF}$ filters like width and range are taken as 7 and [0.1 2]. Experiments are performed using both $\mathrm{ABF}$ and $\mathrm{ABF}$ in combination with mathematical morphology operations and the results are shown. Fig 2(a) depicts the noisy image while figures 2(b) and 2(c) represent the filtered image with $\mathrm{ABF}$ and the filtered image with $\mathrm{ABF}$ and morphological operations. These images are also evaluated by variation of structure elements and variation of width and range of the filter [16]. The dimensions of the structure elements chosen are $3 \times 3,5 \times 5,7 \times 7$ etc. It was identified that best values of filter width of range $(3,7)$ can 
produce good results, otherwise the edges tend to be blurred. Here, the method is numerically performance sensitive with small variation in the parameters and large variations of image
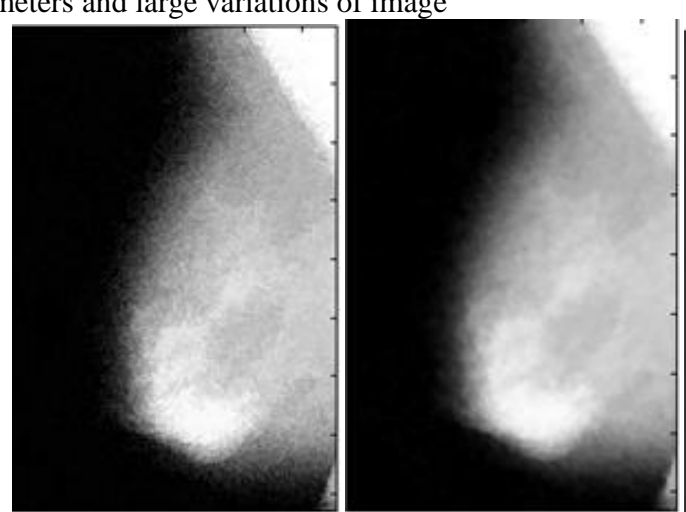

gray values. Sometimes the image gets blurred and takes more time for simulation.

Fig 2: mammogram1 (a) input image (b) ABF output C) ABF with MM

As shown in the fig 3 high dense Mammograms (mammogram2) are the complex images with a large numbers of clusters having a regional maximum. These are the calcium deposits in the breast tissue. In order to detect Micro calcifications we have to evaluate the dynamics of each regional maximum and apply the filtering process. The
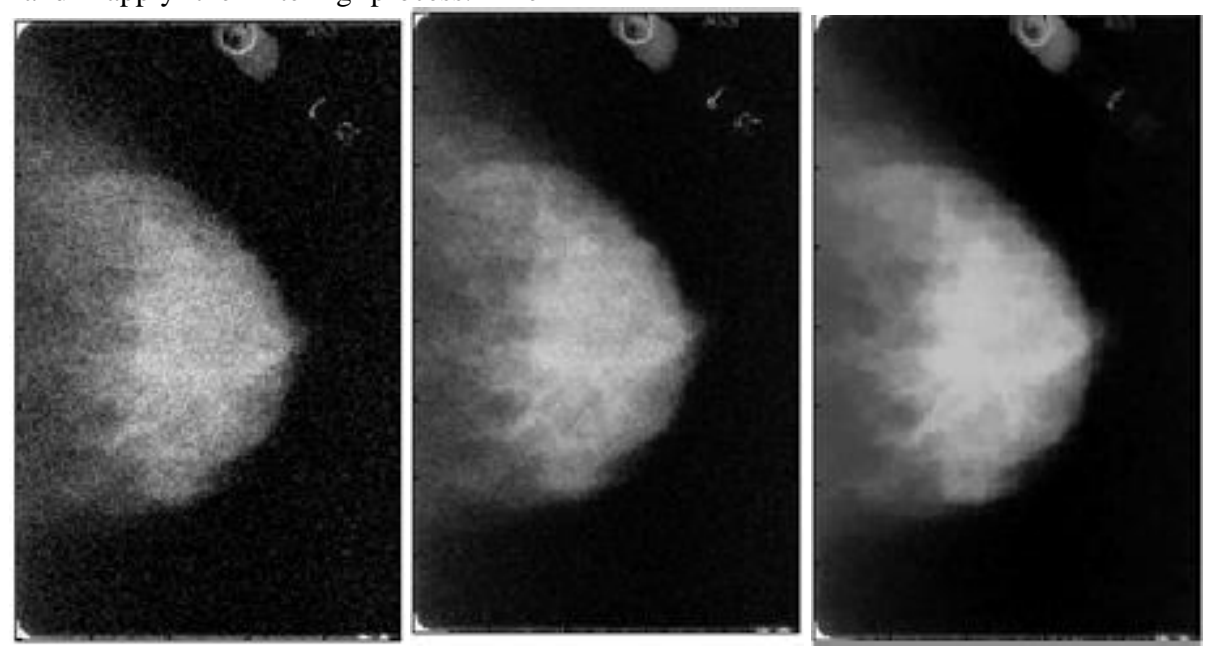

Fig 3: mammogram2 (a) input image (b) ABF output C) ABF with MM

The lesions shown in fig: 4 are small in size, distributed throughout the mammograms [19] and appear to be with low intensity and drastic variations of the contrast. Here it is required to retain the regional maxima and dynamic minima representation of dynamics of a regional maximum is a connected set of pixels with constant gray level value, from which it is difficult to reach a point with higher quality. But the proposed method showed outmost results.
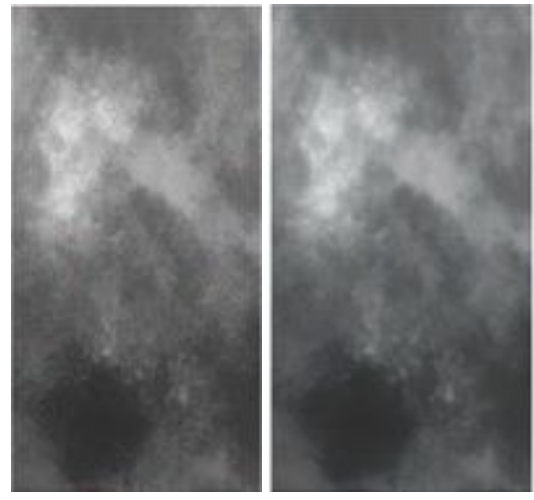

and minimize the noise. The proposed method generated best results as shown in fig 4 . Where Fig.4(a) represent the input image while figures $4(\mathrm{~b})$ and $(4 \mathrm{c})$ represent the $\mathrm{ABF}$ filtered and $\mathrm{ABF}$ filtered with $\mathrm{MM}$ images.

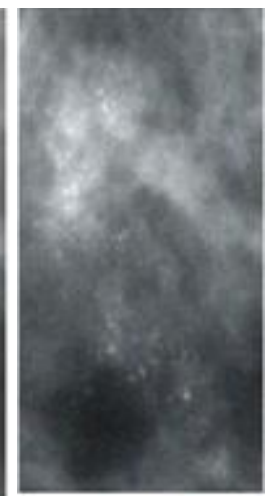

Fig 4: Micro calcifications (a) input image (b) ABF output C) ABF with MM 
Fig 5 shows a part of MRI of brain explored from a volume of data by extracting slices through a three-dimensional MRI data set [21]. There are 27 horizontal slices of dimensions $128 \times 128$ obtained from an MRI data scan of a human cranium. The image is having low intensity gray values. Generally these types of image are generated with noises and the noise being exhibited at low frequency gray level. The proposed method has efficiently filtered the random noise and the output is shown in fig $4(\mathrm{C})$
The lung cancer is diagnosed with chest radiography (x-ray) image. However $\mathrm{x}$-rays are the high frequency values, It generated multi levels of noises like salt and pepper noise, Random noise etc. Removal of these combinational noises is a difficult task. This therefore creates problems in cancer analysis and also percentage of the estimation of cancer being affected [14]. A lung noised image shown in fig $6 \mathrm{~A}$ : is subjected to the proposed method, It is able to efficiently remove the noise parts super imposed on the image, which is shown in fig: $6(\mathrm{C})$.
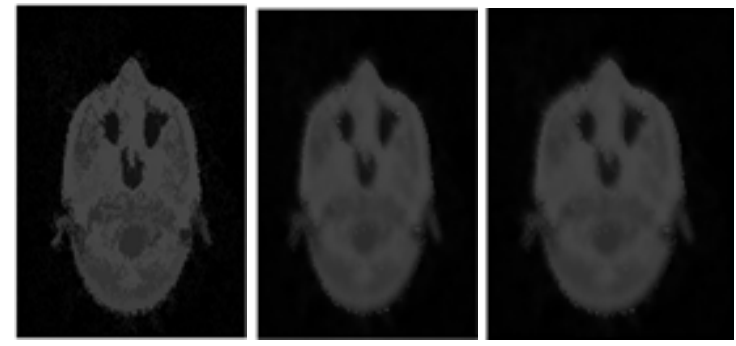

Fig 5: MRI (a) input image (b) ABF output C) ABF with MM
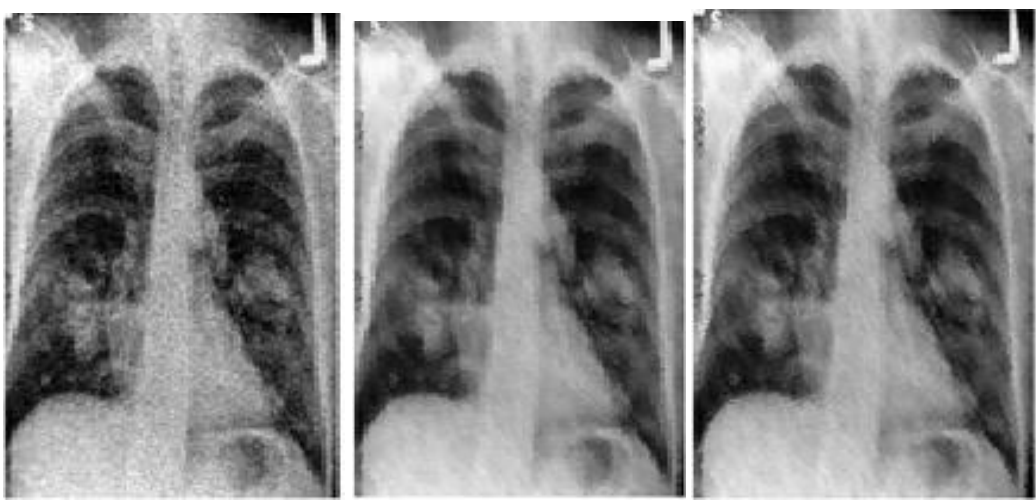

Fig 6: $M R I(a)$ input image (b) $A B F$ output $C$ ) $A B F$ with $M M$

\subsection{Quantitative Evaluation}

In order to get the best results of the proposed method on the basis of the visual appearance of the output image, here we have chosen structure element type, dimensions, window width, and range of the ADF filter. The value also chosen on trial and error basis using human estimation for best suited medical image analysis to get the crucial information from it.

Other features of a de noised image may be measured to quantify the accomplishment of the de noising method. These features include peak signal-to-noise ratio (PSNR), mean square error (MSE), max error, and L2-error. Stopping time $[9,12]$ etc.

\subsubsection{Mean Square Error}

MSE is a risk function, corresponding to the expected value of the squared error loss or quadratic loss. MSE measures the average of the squares of the "errors." The error is the amount by which the value implied by the estimator differs from the quantity to be estimated. The difference occurs because of randomness or because the estimator doesn't account for an information that could produce more accurate estimate. [9].

The MSE is the second moment (about the origin) of the error, which incorporates both the variance of estimator and its bias. For an unbiased estimator, MSE is the variance. Like the variance, MSE has the same units of measurement as the square of the quantity being estimated.

The MSE of an estimator $\alpha_{1}$ with respect to the estimated parameter $\alpha 1$ is defined as

$$
\operatorname{MSE}\left(\alpha_{1}\right)=\mathrm{E}\left[\left(\alpha_{1}-\alpha\right)^{2}\right]
$$

MSE is equal to the sum of the variance and the squared bias of the estimator

$$
\operatorname{MSE}(\alpha 1)=\operatorname{Var}\left(\alpha_{1}\right)+\left(\left(\operatorname{Bias}\left(\alpha_{1}, \alpha\right)\right)^{2}(8)\right.
$$

Thus the quality of an estimator is assessed in terms of its variation and un biasedness.MSE is not equivalent to the expected value of the absolute error. Since MSE is an expectation, it is not a random variable. It may be a function of the unknown parameter $\alpha_{1}$, but it does not depend on any random quantities. However, when MSE is computed for a particular estimator of $\alpha_{1}$ the true value of which is not known, it will be subject to estimation error. In a Bayesian sense, there are cases in which it may be treated as a random variable.

\subsubsection{Peak Signal to Noise Ratio}

The peak signal-to-noise ratio (PSNR) is the ratio between a signal's maximum power and the power of the signal's noise. Signals can have a wide dynamic range, so PSNR is usually expressed in decibels, which is a logarithmic scale $[10,11]$. It is most easily defined via the mean squared error (MSE) 
which for two $\mathbf{m} \times \mathbf{n}$ dimensions monochrome images $\mathrm{I}$ and $\mathrm{K}$ where one of the images is considered a noisy approximation of the other and is defined as:

$$
\mathrm{MSE}=1 / \mathrm{mn} \sum_{i=0}^{m-1} \sum_{j=0}^{n-1}[I(i, j)-K(i, j)]^{2}
$$

The PSNR is defined as:

$$
\text { PSNR=10 LOG }\left(\mathrm{MAX}_{\mathrm{I}} / \mathrm{MSE}\right)
$$

Here, $\mathrm{MAX}_{\mathrm{I}}$ is the maximum possible pixel value of the image. All of these metrics measures a difference between the de noised image and the original clean image. Using these metrics the performance of the proposed method is observed comparing with existing method that is adaptive bilateral filters. A stopping time could be determined by optimizing one of these quantities (as is often done). Furthermore, these metric need not necessarily require an optimum values.

Table 1 lists the PSNR, MSE, MAX-error and L2-error, stopping time for mammogram1 mammogram2, micro calcifications of lesion, MRI and lung x-ray images using adaptive bilateral filters with an $30 \%$ noise is added at the input of the filter.

Table 1: performance metric for adaptive bilateral filter

\begin{tabular}{|c|c|c|c|c|c|}
\hline $\begin{array}{l}\text { S.N } \\
\mathbf{0}\end{array}$ & IMAGE & $\begin{array}{l}\text { PSNR } \\
(\mathbf{d B})\end{array}$ & $\begin{array}{l}\text { MSE } \\
(\mathbf{X} \\
\left.\mathbf{1 0}^{-4}\right)\end{array}$ & $\begin{array}{l}\text { MAX } \\
\text { ERR }\end{array}$ & $\begin{array}{l}\text { TIME } \\
(\mathbf{s})\end{array}$ \\
\hline 1 & $\begin{array}{c}\text { MAMMOGR } \\
\text { AM1 }\end{array}$ & 76.3934 & 15 & 0.2498 & 12.207 \\
\hline 2 & $\begin{array}{c}\text { MAMMOGR } \\
\text { AM2 }\end{array}$ & 77.2993 & 12 & 0.3982 & 35.302 \\
\hline 3 & $\begin{array}{c}\text { Micro } \\
\text { lesions }\end{array}$ & 74.15 & 25 & 0.3453 & 70.5 \\
\hline 4 & MRI & 71.948 & 36 & 0.3265 & 3.41 \\
\hline 5 & Lung & 73.03 & 32 & 0.3878 & 6.15 \\
\hline
\end{tabular}

Table 2 lists the PSNR, MSE, MAX-error and L2-error, stopping time for mammogram1 mammogram2, micro calcifications of lesion, MRI and lung x-ray images using proposed method with a $30 \%$ noise are added at the input of the filter. It is observed that by including the mathematical morphological operations the performance levels in all these metrics are enhanced nearly $10 \%$.

Table2: performance metric for Morphological adaptive bilateral filter with structure elements- 'disc' dimensions-3 $\mathbf{x} 3$.

\begin{tabular}{|c|l|l|l|l|l|}
\hline $\begin{array}{l}\text { S.N } \\
\mathbf{o}\end{array}$ & IMAGE & $\begin{array}{l}\text { PSNR } \\
(\mathbf{d B})\end{array}$ & $\begin{array}{l}\text { MSE } \\
(\mathbf{X} \\
\left.\mathbf{1 0}^{-4}\right)\end{array}$ & $\begin{array}{l}\text { MAXE } \\
\mathbf{R R}\end{array}$ & $\begin{array}{l}\text { TIME } \\
(\mathbf{s})\end{array}$ \\
\hline 1 & $\begin{array}{c}\text { MAMMOG } \\
\text { RAM1 }\end{array}$ & 79.142 & 7.922 & 0.4092 & 11.752 \\
\hline 2 & MAMMOG & 80.1973 & 6.213 & 0.5676 & 34.135 \\
\hline
\end{tabular}

\begin{tabular}{|c|c|c|c|c|c|}
\hline & RAM2 & & & & \\
\hline 3 & $\begin{array}{c}\text { Micro } \\
\text { lesions }\end{array}$ & 75.439 & 17 & 0.3391 & 68.751 \\
\hline 4 & MRI & 72.453 & 34 & 0.2957 & 3.392 \\
\hline 5 & Lung & 79.3693 & 7.518 & 0.2985 & 5.826 \\
\hline
\end{tabular}

For example consider the mammogram image shown in fig 2 , it generates the PSNR value for $\mathrm{ABF}$ of $76.33 \mathrm{~dB}$, using $\mathrm{ABF}$ with MM the value becomes $79.11 \mathrm{~dB}$. Similarly the MSE value $15 \times 10^{-4}$ of $A B F$., where as for $A B F$ with $M M$ the value reduced to $7.922 \times 10^{-4}$. Also stopping time for $\mathrm{ABF}$ $12.202 \mathrm{~s}$ Whereas $\mathrm{ABF}$ with $\mathrm{MM}$ it is reduced to $11.752 \mathrm{~s}$. However, it may not be a good indicator of the best de-noising results, as sharp edges is to be preserved for efficient pre processing technique in CAD (computer aided system) system design for effective removal of noise.

Table 3: is the relative comparison between the design parameters. Those are structure element, dimensions with width of window. Here structure element keeping a fixed value and width is varied in descending steps from 7 to 4 . The structure element is kept at constant dimensions i.e. $3 \times 3$, $5 \times 5,7 \times 7$ and varied the staring with 7 decrement the steps by one up to 4 value is to be experimented and the metric MSE,PSNR, stopping time is calculated.

\begin{tabular}{|c|c|c|c|c|}
\hline S.No & width & $\operatorname{MSE}\left(\mathrm{X} \mathrm{10}^{-4}\right)$ & $\begin{array}{l}\text { PSNR } \\
\text { (dB) }\end{array}$ & TIME(s) \\
\hline \multicolumn{5}{|c|}{ Structure element -Disc dimensions $3 \times 3$} \\
\hline 1 & 7 & 7.94 & 79.131 & 11.633 \\
\hline 2 & 6 & 5.89 & 80.03 & 11.63 \\
\hline 3 & 5 & 5.57 & 80.6712 & 11.633 \\
\hline 4 & 4 & 5.4068 & 80.8014 & 11.633 \\
\hline \multicolumn{5}{|c|}{ Structure element -Disc dimensions $5 \times 5$} \\
\hline 1 & 7 & 11.1 & 77.667 & 11.67 \\
\hline 2 & 6 & 9.464 & 78.3699 & 11.67 \\
\hline 3 & 5 & 9.3 & 78.4081 & 11.67 \\
\hline 4 & 4 & 8.92 & 78.59 & 11.67 \\
\hline \multicolumn{5}{|c|}{ Structure element -Disc dimensions $7 \times 7$} \\
\hline 1 & 7 & 19 & 75.254 & 11.7 \\
\hline 2 & 6 & 15 & 76.4463 & 11.7 \\
\hline 3 & 5 & 14.8 & 76.48 & 11.7 \\
\hline 4 & 4 & 14.1 & 76.58 & 11.7 \\
\hline
\end{tabular}

Table: 3 shows the relation between the design parameters 
Also it was observed that if the width of window exceed the range [4 7] or structure element chosen is other than a disc or is exceeding the dimensions more than the $7 \times 7$ the output image is getting blurred. Fig. 7 shows the estimated width of filter versus MSE with structure element dimensions. It is observed that the lowest dimension of SE i.e 3 X 3 has given the minimum MSE value with a given window width of the filter.

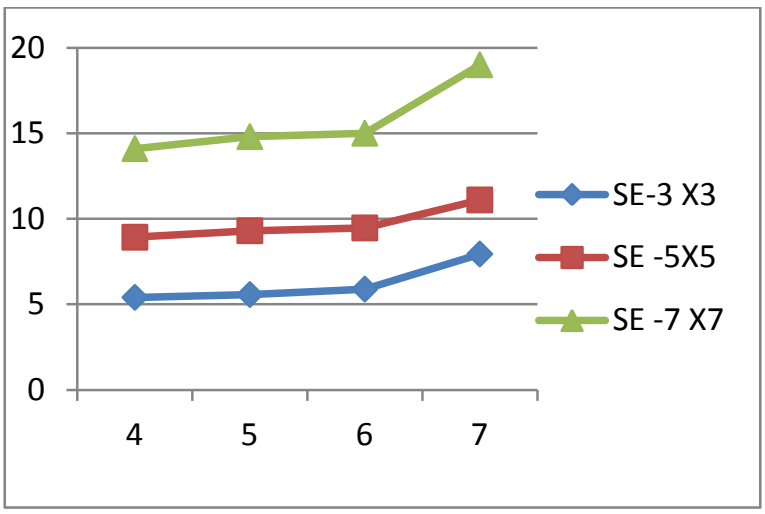

Fig7: MSE VS WIDTH OF ABF FILTER

Fig. 8 shows the estimated width of the filter versus PSNR with a structure element dimension. It is observed that the lowest dimension of SE i.e $3 \times 3$ has given the high PSNR value with given window width of the filter

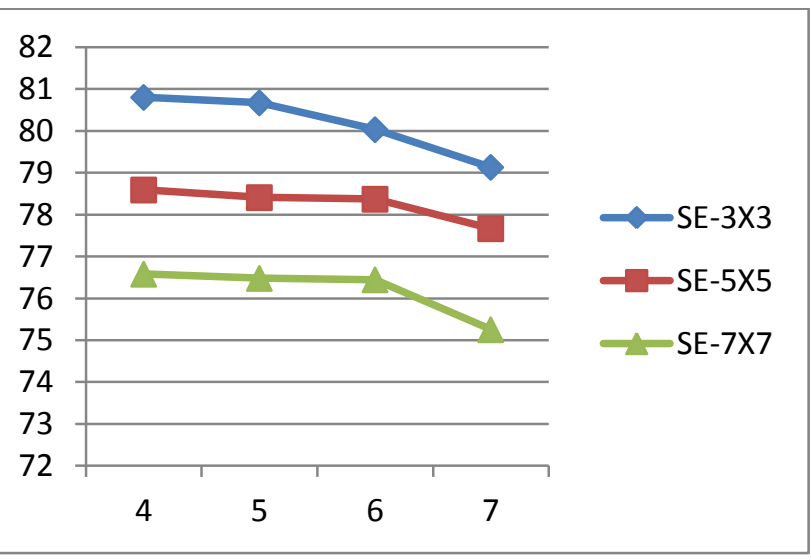

Fig 8: PSNR VS WIDTH OF ABF FILTER

Fig. 9 shows the estimated structure element dimensions versus PSNR with a given width value of the filter. It is observed that the lowest dimension of SE that is $3 \mathrm{X} 3$ and the least value of the window function(4) has given the high PSNR value with given window width of the filter.

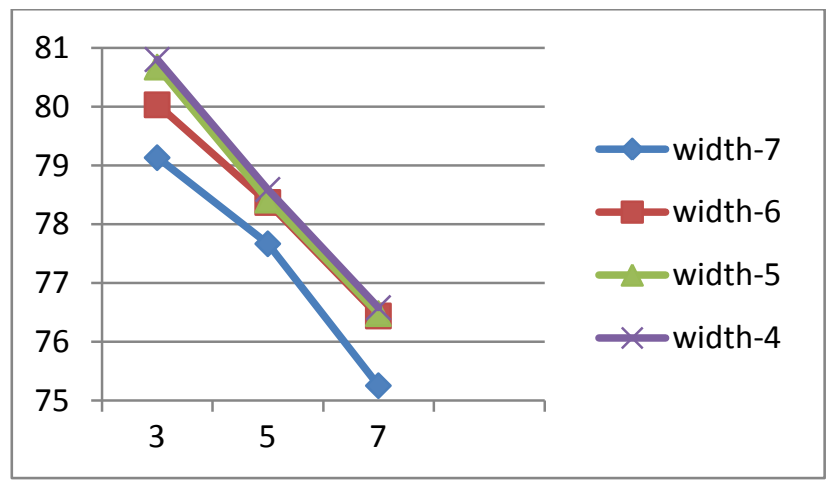

Fig. 9: PSNR VS structure element dimensions
The proposed method was applied to medical images with different standard deviation noises. The qualitative metrics of the filters at various design parameters of the resultant image was measured for the proposed method, the adaptive bilateral filtering method and the state-of-the-art image de-noising method using Gaussian scale mixtures proposed by A.wang. et.al $[2,12]$ proposed method for comparison. As Shown in fig:10 The MSE value is reduced using MM operators with combination of $\mathrm{ABF}$.

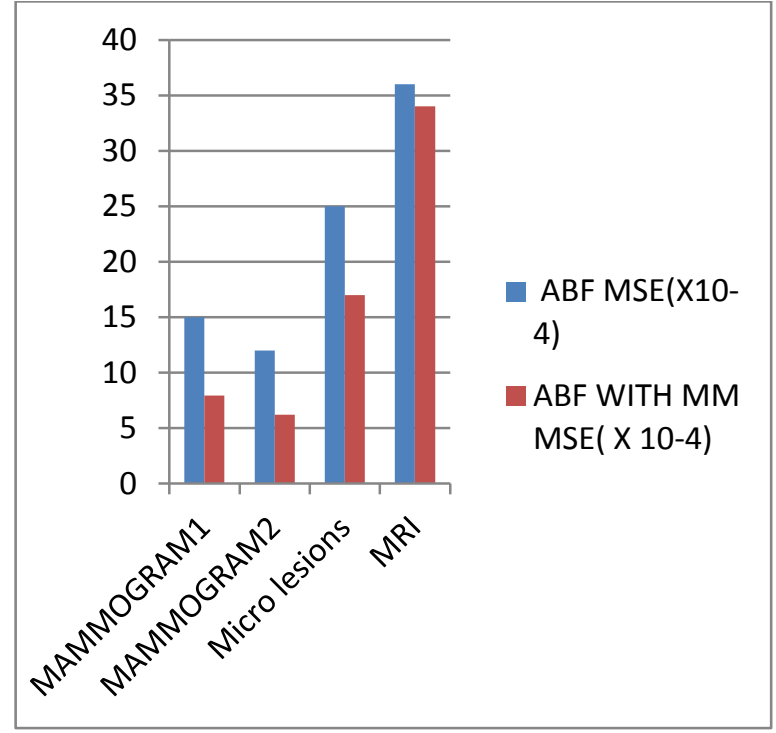

Fig:10 A comparison of $\mathrm{ABF}$ and $\mathrm{ABF}$ with $\mathrm{MM}$ w.r.t. MSE

A summary of the results states that the average PSNR is around $75 \mathrm{~dB}$ by Adaptive bilateral filter. As shown in the fig 11 show the enhanced PSNR value by using MM operators with ABF The proposed method achieves noticeable PSNR gains over $81 \mathrm{~dB}$ when algorithm is simulated with MATLAB 13.

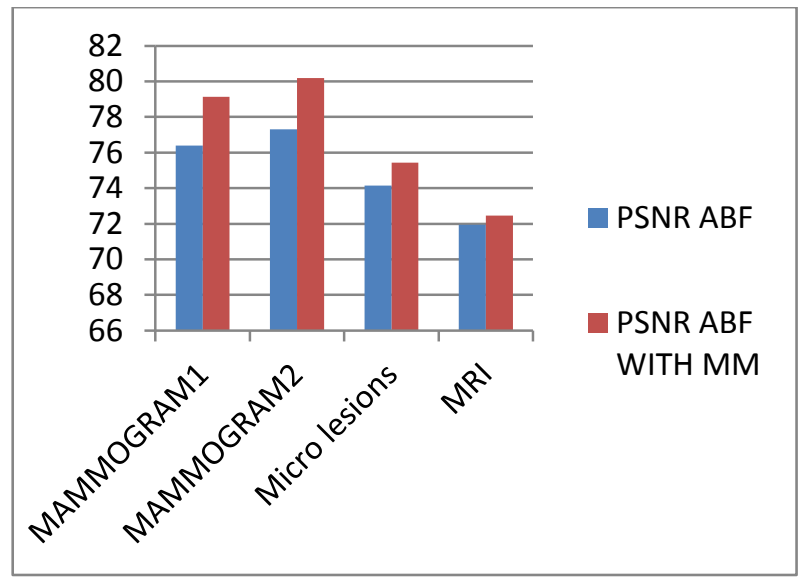

Fig:11 A comparison of $\mathrm{ABF}$ and $\mathrm{ABF}$ with $\mathrm{MM}$ w.r.t. PSNR

It is observed that the proposed method produced a noise removal image signal with noticeably improved perceptual quality compared to the Wang and the original bilateral filtering method. 


\section{CONCLUSIONS}

The adaptive morphological bilateral filter sharpens a medical image without any loss of information. It is increasing the slope of the edges without producing overshoot or undershoots. In the adaptive bilateral filter, the edge slope is enhanced by transforming the histogram via a range filter with adaptive offset and width. It is also proved that mathematical morphological operation increases the efficiency of the filters and the quality appearance of the output medical images, which was experimented on various types of medical images. Design parameters are compared with quality metrics. The main limitation is, this method is not much effective on the image having the corner edges. Future development of this method is to be modified by an addition of frequency domain operations along with proposed method.

\section{ACKNOWLEDGEMENTS}

This research work is fully funded and support by our institutions. The authors show the gratitude to the institutions, Head of the institutions, Co Faculty, Scholars. The authors thankful to the Local government Hospital is near to our institutions to support their clinical suggestions to progress of this work.

\section{REFERENCES}

[1] Buyue Zhang, Jan P. Allebach 'Adaptive Bilateral Filter for Sharpness Enhancement and Noise Removal', IEEE TRANSACTIONS ON IMAGE PROCESSING, VOL. 17, NO. 5, MAY 2008

[2] Alexander Wong, 'Adaptive bilateral filtering of image signals using local phase characteristics' Elsevier Signal Processing 88 (2008) 1615-1619

[3] Jinshan Tang, Shengwen Guo, Qingling Sun, Youping Deng, Dongfeng Zhou speckal noise reduction by bilateral filter BioComp 2009 Las Vegas, NV, USA. 1316 July 2009

[4] Hyung W. Kang Charles K. Chui Uday K. Chakraborty 'A unified scheme for adaptive stroke-based Rendering' Visual Comput (2006) 22: 814-824

[5] Das and R. M. Rangayyan, "Enhancement of image edge sharpness and acutance," in Proc. SPIE, 1997, vol. 3026, pp. $133-142$

[6] Yoshinori ITO, Takanori SATO, Noritaka YAMASHITA, Jianming LU, Hiroo SEKIYA and Takashi YAHAGI IEEE 4261 ISCAS 2006

[7] Xiaoping Lin 'Research and Application of Mathematical Morphology Algorithms' on OSSC IEEE- OSSC 2009 173Joseph M. Reinhardt and William E. Higgins 'Efficient Morphological Shape Representation' IEEE TRANSACTIONS ON IMAGE PROCESSING, VOL. 5, NO. 1, JANUARY 199689

[8] Joseph M. Reinhardt and William E. Higgins 'Efficient Morphological Shape Representation' IEEE Transactions On Image Processing, Vol. 5, No. 1, January 199689.
[9] Patrick Guidotti - Kate Longo 'Two Enhanced Fourth Order Diffusion Models for Image Denoising' J Math Imaging Vis (2011) 40: 188-198

[10] S. Kim and J. P. Allebach, "Optimal unsharp mask for image sharpening and noise removal," J Electron Imaging, vol. 14, no. 2, pp. 023007-1 - 023007-13, 2005.

[11] .Rafael C. Gonzalez, Richard E. Woods and Steven L. Eddins (2004).' Digital Image Processing using MATLAB. Pearson Education' ISBN 978-81-7758-8989.

[12] B.Sridhar \& Dr.K.V.V.S.Reddy 'Performance Evaluation of 2D Adaptive Bilateral Filter For Removal of Noise From Robust Images', International Journal of Image Processing (IJIP), Volume (7): Issue (1): 201338.

[13] Frank Y. Shih Vijayalakshmi Gaddipati, 'General sweep mathematical morphology' Pattern Recognition 36 (2003) $1489-1500$

[14] Jinshan Tang 'A multi-direction GVF snake for the segmentation of skin cancer images' Pattern Recognition 42 (2009) 1172 - 1179.

[15] Stelios Halkiotis 'Automatic detection of clustered micro calcifications' in digital mammograms using mathematical morphology and neural networks Signal Processing 87 (2007) 1559-1568.

[16] P. Salembier and J. Serra, 'Flat Zones Filtering, Connected Operators, and Filters by Reconstruction', IEEE TRANSACTIONS ON IMAGE PROCESSING, Vol. 4, NO. 8, AUGUST 1995.

[17] Ghassan Hamarneh and Judith Hradsky Bilateral Filtering of Diffusion Tensor Magnetic Resonance Images, IEEE TRANSACTIONS ON IMAGE PROCESSING, VOL. 16, NO. 10, OCTOBER 2007 2463.

[18] .Joseph M. Reinhardt, Efficient Morphological Shape Representation IEEE transactions on image processing, VOL. 5, NO. 1, JANUARY 199689.

[19] Berkman Sahiner, Computer-Aided Characterization of Mammographic Masses: Accuracy of Mass Segmentation and Its Effects on Characterization, IEEE transactions on medical imaging, VOL.20, NO. 12, DECEMBER 20011275

[20] Zijuan Yu, Julian Zhao Research Advances and Prospects of Mathematical Morphology in Image Processing CIS 20081242

[21] Benoit Scherer Distributed Local MRF Models for Tissue and Structure Brain Segmentation IEEE transactions on medical imaging, VOL. 28, NO. 8, AUGUST 2009. 